\title{
Integral Adaptive Control For Uncertain System Based On Wrong High Order System Information
}

\author{
Wenguang Zhang ${ }^{1, a}$, Lingling Wang ${ }^{1}$ and Tian Lan $^{2}$ \\ ${ }^{1}$ Department of control engineering, Naval aeronautical and astronautical university, Yantai of China, \\ 264001 \\ ${ }^{2}$ Department of strategy and campaign, Naval command college, Nanjing of China, 264001 \\ azwg1024@126.com
}

Keywords: Integral control, adaptive control, uncertain system, stability, wrong information

\begin{abstract}
In this paper, an adaptive control design and stability analysis for a class of uncertain systems with unknown models is carried out. In particular, the adaptive control effect analysis is carried out based on the assumption that the unknown system mode is estimated with the wrong mode information. Based on the wrong higher order mode function and the wrong low order mode function, the detailed simulation analysis is carried out, which verifies the correctness of the existence of the dead zone.
\end{abstract}

\section{Introduction}

System uncertainty is a common problem in control engineering[1-5]. Adaptive method is one of the commonly used methods to deal with uncertainty, and it is one of the more effective methods at present. If the uncertainty of the system can be described as a multiplicative form of the unknown parameters and the known modes[6-10], the algorithm of adaptive parameter estimation has been very mature. But if the system model is unknown, namely, the existence of unknown nonlinear functions and the nonlinear function of order information is unknown or even false information, then how to analysis the role of adaptive control? This problem at present, there are few literatures actually related with the problem, which is a problem frequently encountered in actual control. This paper will be based on the system of unknown and wrong model function information, then we use high order or low order wrong mode to design adaptive controller, and discussed the problem that how to analyze control effect. In this paper, the specific theoretical analysis of these two kinds of special circumstances and the correctness of the conclusion of this paper is verified by the final simulation analysis.

\section{Model Description}

Consider the bellow first system

$$
\dot{x}=f(x)+u
$$

Where $f(x)=a_{1} x+a_{2} \sin x+a_{3} x^{2}$, and $f(x)$ not only contains unknown parameters and also the mode function is also unknown.

And the control object is to design a PID control law such that the state can converged to expected value $x^{d}$, where $\dot{x}^{d}=0$.

And we assume that we made a wrong estimation of system mode function and we assume there exist a high order mode $x^{3}$ and in fact the system does not exist that mode.

\section{Analysis Of Integral Control Effect For System With Unknown Mode Function}

Define a error variable as

$$
z=x-x^{d}
$$

Then 
$\dot{z}=\dot{x}=a_{0} x^{1 / 3}+a_{1} x+a_{2} \sin x+a_{3} x^{2}+u$

According to the wrong estimation of system mode function, we design a high order mode adaptive control law as

$u=-\hat{a}_{3} x^{3}-k_{1} z-k_{2} \int z d t-k_{3} \operatorname{sign}(z)$

And choose a Lyapunov function as

$V=\frac{1}{2} z^{2}+\frac{1}{2 \Gamma} \tilde{a}_{3}^{2}+\frac{k_{2}}{2}\left(\int z d t\right)^{2}$

Where $\tilde{a}_{3}=0-\hat{a}_{3}$, then

$\dot{\tilde{a}}_{3}=-\dot{\hat{a}}_{3}$

And solve the derivative of Lyapunov function as

$\dot{V}=z \dot{z}+\frac{1}{\Gamma} \tilde{a}_{3} \dot{\tilde{a}}_{3}+k_{2} z \int z d t$

Consider that

$\dot{z}=\tilde{a}_{3} x^{3}+a_{3} x^{2}+a_{1} x+a_{2} \sin x+a_{0} x^{1 / 3}-k_{1} z-k_{2} \int z d t$

Then

$$
z \dot{z}=z \tilde{a}_{3} x^{3}+a_{3} x^{2} z+a_{1} x z+a_{2} z \sin x+a_{0} z x^{1 / 3}-k_{1} z z-k_{2} z \int z d t-k_{3}|z|
$$

so

$z \dot{z}+k_{2} z \int z d t=z \tilde{a}_{3} x^{3}+a_{3} x^{2} z+a_{1} x z+a_{2} z \sin x+a_{0} z x^{1 / 3}-k_{1} z z-k_{3}|z|$

And design the turning law as

$\dot{\tilde{a}}_{3}=-\dot{\hat{a}}_{3}=-\Gamma z x^{3}$

then

$-\frac{\dot{\tilde{a}}_{3}}{\Gamma}=z x^{3}$

so

$z \dot{z}+k_{2} z \int z d t=-\frac{\dot{\tilde{a}}}{\Gamma} \tilde{a}_{3}+a_{3} x^{2} z+a_{1} x z+a_{2} z \sin x+a_{0} z x^{1 / 3}-k_{1} z z-k_{3}|z|$

then

$z \dot{Z}+\frac{\dot{\tilde{a}}_{3}}{\Gamma} \tilde{a}_{3}+k_{2} z \int z d t=a_{1} x z+a_{2} z \sin x+a_{0} z x^{1 / 3}+a_{3} x^{2} z-k_{1} z z-k_{3}|z|$

And the identification law of unknown parameter is designed as

$\dot{\hat{a}}_{3}=\Gamma z x^{3}$

Then

$\dot{V}=z \dot{z}+\frac{\dot{\tilde{a}}_{3}}{\Gamma} \tilde{a}_{3}+k_{2} z \int z d t=a_{1} x z+a_{2} z \sin x+a_{0} z x^{1 / 3}+a_{3} x^{2} z-k_{1} z z-k_{3}|z|$

And considering the finite range stable characteristic of nonlinear system, we assume there exists bounded $D$ and $D_{1}$ such that $|x|<D, a_{1} x z+a_{2} z \sin x+a_{0} z x^{1 / 3}+a_{3} x^{2} z<D_{1}|z|$, then there exists a big gain $k_{3}$ such that

$$
\dot{V} \leq-k_{1} z z \leq 0
$$

So the system can be stable is the initial condition is bounded.

Also if we made a wrong estimation for the mode function of system and we think the system has a unknown mode function $x^{1 / 9}$ which is not exist in the real system in fact.

Then we can design a adaptive control law as

$$
u=-\hat{a}_{3} x^{1 / 3}-k_{1} z-k_{2} \int z d t-k_{3} \operatorname{sign}(z)
$$

And design the adaptive law as 


$$
\dot{\hat{a}}_{3}=\Gamma z x^{1 / 9}
$$

So main analysis process and proof are the same as the first situation, and the same conclusion can be made.

\section{Simulation Analysis}

Based on above system and we set $f(x)=a_{0} x^{1 / 3}+a_{1} x+a_{2} \sin x+a_{3} x^{2}$ where parameters $a_{j}=j+1,(j=0,1,2,3)$, and a kind of soft function is used to take place of sign function, so the high order power control program can be constructed with simulink program as bellows:

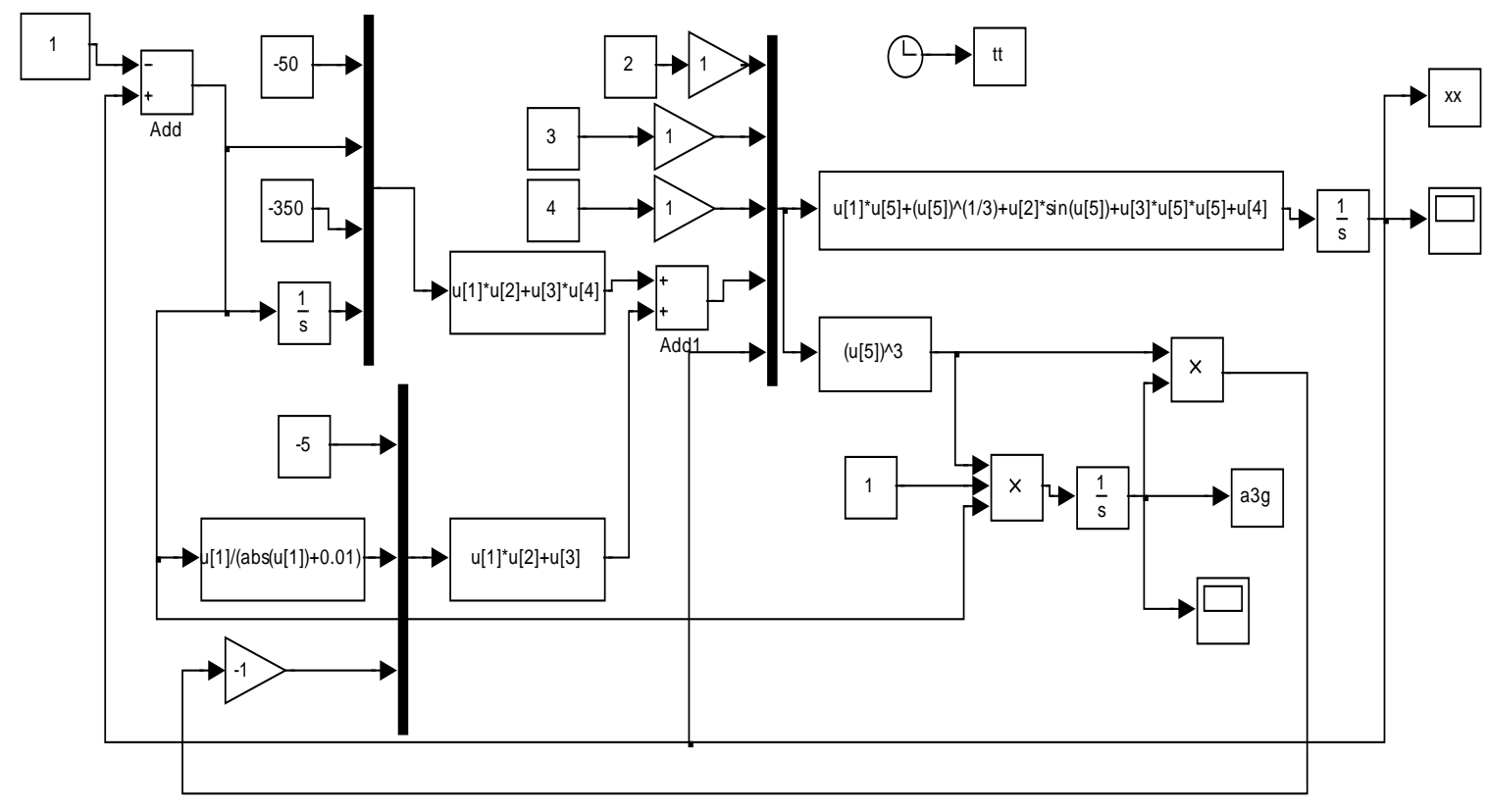

Fig.1. The simulink structure of program

And the simulation result can see below figure 2.

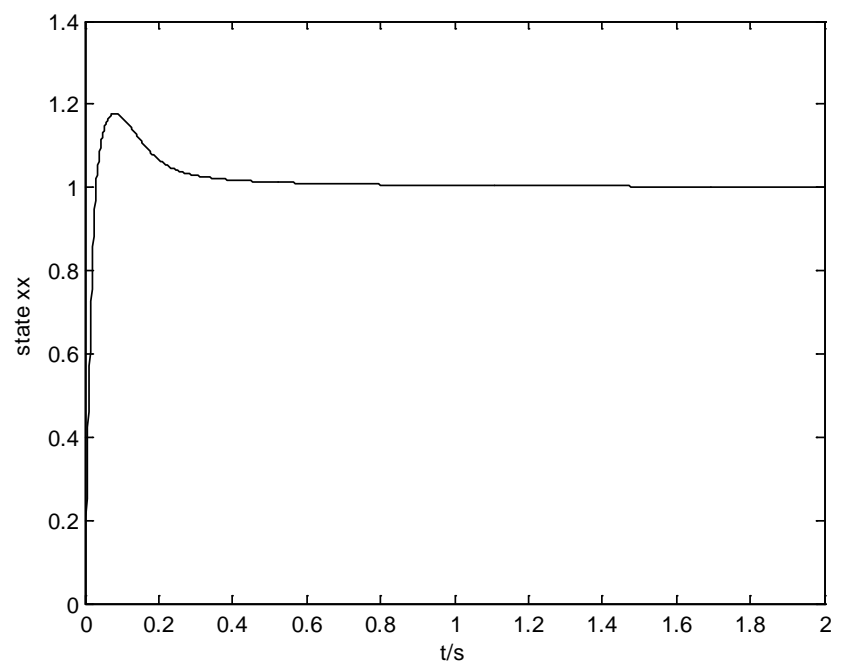

Fig.2. The curve of system state 


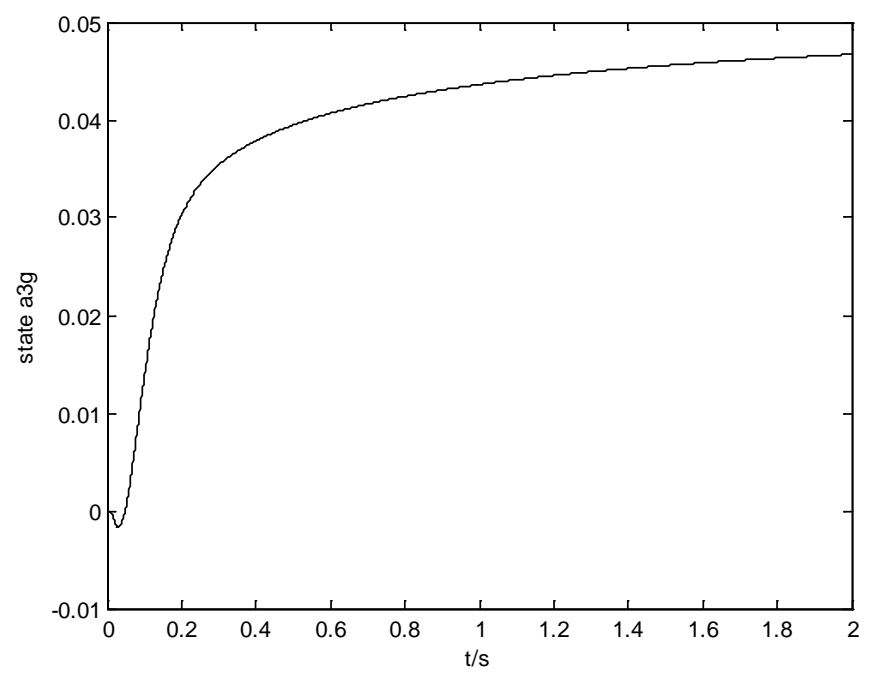

Fig.3. The estimation of unknown parameter

And if we choose a small signal to do the simulation, set $x^{d}=0.0001$, then it can be found from the simulation result that the signal can be traced accurately although a long time is needed.

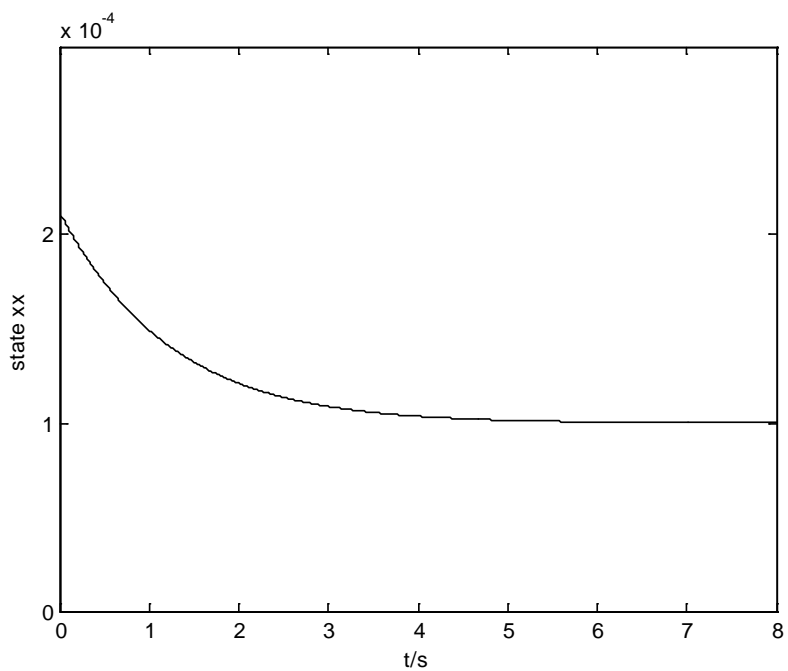

Fig.4. The estimation of system state

And the system input signal can be reduced as $x^{d}=0.000001$, and the control gain is not changed, then there is a dead zone phenomenon which can see below figure 5 .

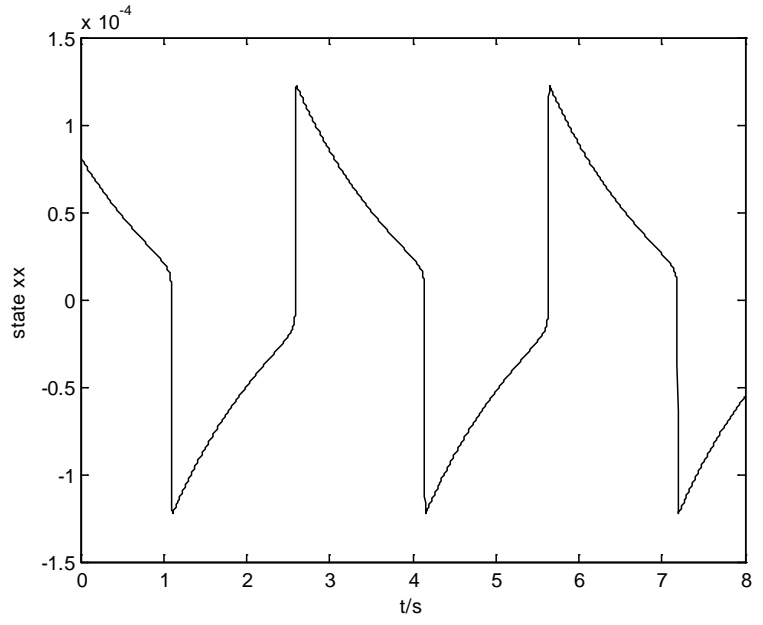

Fig.5. The phenomenon of dead zone 


\section{Conclusion}

This paper analyzes the practical significance of adaptive control for a class of complex situations, that is, if the unknown mode information is wrong, how to design and analyze the impact of adaptive control system performance? Respectively, for the wrong high order modes and wrong low order models which is not existed, the adaptive design control effect is analyzed, we come to the conclusion that the dead zone is always exist. Finally, the detailed simulation analysis, also shows the correctness of the conclusion of the thesis.

\section{References}

[1] J.S. Uhlman, N.E.Fine, D.C. Kring. Calculation of the Added Mass and Damping Forces on Supercavitating Bodies. The 4th International Symposium on Cavitation,California,2001:7 13

[2] D. Clarke. Calculation of the Added Mass of Elliptical Cylinders in Shallow Water. Ocean Engineering.2001,28(4):61 72

[3] C.J.Atkinson, R.G. Urso. Modeling of Apparent Mass Effects for the Real-Time Simulation of a Hybird Airship. AIAA Modeling and Simulation Technologies Conference and Exhibit,Keystone.2006:21 32

[4] Yokomaku Y. The Stratospheric Platform Airship R\&D Program of Japan. The 2nd Stratospheric Platform Systems Workshop, Tokyo Japan.2000:7 13

[5] S.P. Jones, J.D. Laurier. Aerodynamic Estimation Techniques for Aerostats and Airships. AIAA Lighter-than-Air Systems Conference,Annapolis,2004: 88 94

[6] M.T. Soylemeza, N. Munrob, H. Bakic. Fast Calculation of Stabilizing PID Controllers. Automatic, 2003,39(7):121 126

[7] Etkin B, Theory of the flight of Airplanes in Isotropic Turbulence Review an Extension, AGARD Rept.1961:372

[8] David K. Schmidt, James Stevens, Jason Roney. Dynamic Modeling, Control, and Station-Keeping Guidance of A Large High-Altitude "Near-Space" Airship. AIAA Guidance, Navigation, and Control Conference and Exhibit. 2006-6781

[9] David K. Schmidt. Modeling and Near-Space Station-Keeping Control of a Large High-Altitude Airship. Journal of Control and Dynamics,2007,30(2):540 547

[10] Azinheira, J.R., A. Moutinho, Hover Control of an UAV With Backstepping Design Including Input Saturations. IEEE Transactions on Control System Technology, 2008.16(3):517-526 